\title{
ÉTUDE SUR LES HYBRIDES DES BIOTYPES DE CULEX PIPIENS LINNÉ
}

\author{
Par J. CALLOT
}

L'hybridation entre les sous-espèces qui composent l'espèce linnéenne Culex pipiens a déjà donné lieu à différents travaux, en particulier ceux de Roubaud et de Weyer en Europe, de Farid, de Sunderaraman, de Rozeboom et Gilford en Amérique. Personnellement, j'ai donné des résultats concernant les biotypes européens en 1947 et en 1954 .

Mais, à part celles qui sont consignées dans ma note de 1947, ces différentes recherches n'intéressent que la morphologie de l'adulte ou quelques points du comportement.

Dans la présente note, je voudrais indiquer le résultat du croisement entre les sous-espèces européennes et Culex pipiens fatigans d'Afrique, en insistant plus particulièrement sur la morphologie larvaire.

\section{Matériel}

J'ai utilisé pour ces recherches, ou pour des comparaisons, une souche de Culex pipiens autogenicus que j'entretiens au laboratoire, une souche de Culex pipiens fatigans originaire d'Afrique Occidentale (1), enfin des Culex pipiens pipiens provenant de pontes trouvées dans la nature ou dans des pièges à ponte.

\section{$1^{\circ}$ Garactères des autogènes}

Cette souche, avec laquelle ont été réalisés les croisements ou les tentatives de croisements, a pour origine une femelle ayant piqué l'homme dans un immeuble de Strasbourg. Les adultes ont les caractères classiques des autogènes, avec une légère ponctuation sternale (sternopunctatus ?), la trompe du mâle dépasse l'extrémité distale du quatrième article du palpe dans $95 \%$ des cas; ils sont fortement sténogames, puisque l'accouplement a lieu dans des tubes

(1) Je la dois à l'obligeance de M. E. Abonnenc, que je remercie vivement pour sa complaisance.

Ann. de Parasitologie, T. XXX, $\mathrm{N}^{\circ} 4 .-1955$. 


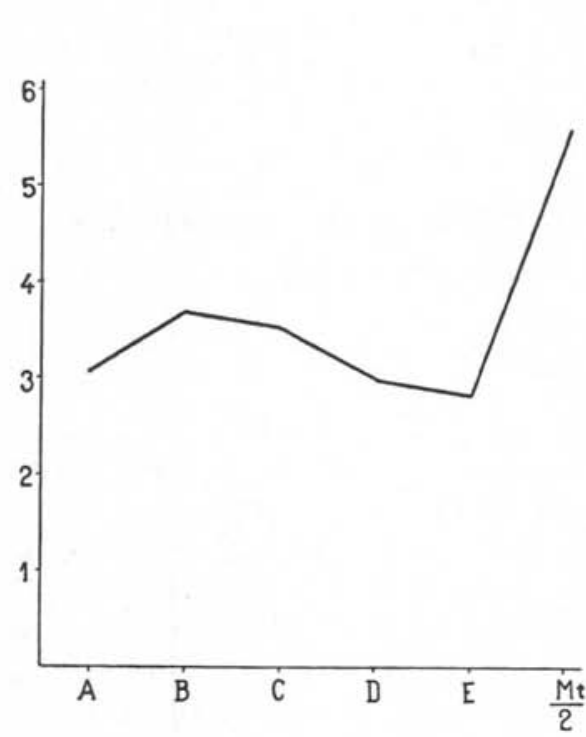

de $40 \times 15 \mathrm{~mm}$. L'autogénèse se manifeste, au moins à l'examen des ovaires par dissection, chez près de $100 \%$ des femelles si la nourriture larvaire a été suffisante.

Cette souche entretenue dans mon laboratoire depuis l'été de 1952 ne présente au cours des générations que peu de variations de ses caractères biométriques larvaires.

Les indices empioyés sont établis sur les exuvies des larves au quatrième stade; je les ai déjà utilisés pour mes recherches publiées en FIt. 1. - Culex pipiens autogenicus (Strasbourg) 1947. Ils sont identiques à ceux proposés par Marshall pour les cinq premiers $(\mathrm{A}, \mathrm{B}$, C, D, E), mais j'ai remplacé les indices obtenus sur les adultes par l'indice des dents du mentum $(\mathrm{Mt}=$ dents de droite + dents de gauche $)$.

2

Ce sont ces indices qui

sont reportés sur les morphogrammes; mais Mt a été réduit de moitié ${ }_{2}^{(\text {Mit) }}$ pour la commodité. Sur les tableaux qui suivent, à côté des moyennes, on a porté l'erreur standard ou écart type de la moyenne : $\mathrm{s}=\frac{\sigma}{\sqrt{\mathrm{n}}}, \sigma$ étant l'écart type $: \sigma=\sqrt{\frac{\Sigma \mathrm{d}^{2}}{\mathrm{n}-1}}$, pour les indices ayant réellement une valeur différente dans les divers biotypes, c'est-à-dire A, B, Mt.

Les caractères biométriques ainsi établis pour les autogènes de Strasbourg utilisés dans les expériences et à ce moment peuvent être résumés dans le tableau I et la figure 1 : 
Tableau 1. - Larves de C. p. autogenicus (Strasbourg)

\begin{tabular}{|c|c|c|c|c|c|c|c|c|c|c|}
\hline \multicolumn{3}{|c|}{ StPHoN } & \multicolumn{6}{|c|}{ SOtES DU SIPHOX } & \multicolumn{2}{|c|}{ Mextum } \\
\hline A & & $\mathbf{s}$ & B & & $\mathrm{s}$ & C & D & $\mathrm{E}$ & Mt & $\mathbf{s}$ \\
\hline 3,07 & \pm & 0,04 & 3,70 & \pm & 0,24 & 3,54 & 3,00 & 2,86 & 11,27 & $\pm 0,20$ \\
\hline
\end{tabular}

Un calcul des moyennes à différentes époques donne pour 238 exemplaires les valeurs suivantes : $\mathrm{A}=3,11 ; \mathrm{B}=3,57$; $\mathrm{Mt}=10,98$.

\section{$2^{\circ}$ Garactères de Culex pipiens fatigans}

Cette souche est issue d'une femelle gorgée provenant de Dakar. Les adultes présentent les caractères classiques des $C$. fatigans, c'est dire combien ils ressemblent (à part l'hypopygium des mâles) à Culex pipiens. Mais leur coloration diffère de celle des autogènes qui sont plus clairs. La coloration des sternites abdominaux est très variable chez les individus issus d'une même ponte : certains exemplaires n'ont aucune marque sombre sternale. La trompe du mâle dépasse, comme chez autogenicus, l'extrémité distale du quatrième article du palpe dans tous les exemplaires que j'ai examinés.

Ils sont sténogames, piquent volontiers l'homme, mais, comme je l'ai constaté aux dépens de certaines expériences, la ponte se fait mieux s'ils sont gorgés sur le poulet. Il n'y a aucune trace d'autogénèse chez les femelles disséquées.

Les larves issues de la première ponte s'élèvent très facilement avec de la poudre de biscuit pour chiens.

Leur morphogramme m'a montré, comme je l'avais déjà constaté sur des lar-

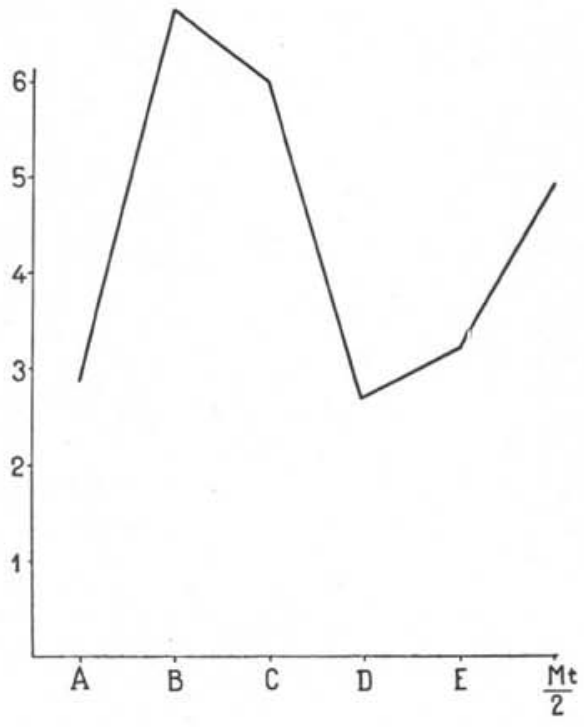

Fı. 2.-- Culex pipiens fatigans (Dakar) 
ves de $C$. fatigans d'Indochine, d'Afrique Occidentale et des Mascareignes, qu'il y a une différence très nette entre celui-ci et celui des autogènes, et à fortiori celui du C. pipiens pipiens. L'indice siphonique sur exuvie est très bas chez fatigans, sans compter même la forme un peu différente du siphon. Le nombre de soies des deux premières touffes ( $\mathrm{B}, \mathrm{C}$ ) est bien plus considérable que chez autogenicus et bien entendu que chez pipiens. Les dents du mentum sont moins nombreuses que chez autogenicus (tab. II et fig. 2).

Tableau II. - Larves de Culex p. faligans de Dakar

\begin{tabular}{|c|c|c|c|c|c|c|c|c|c|c|c|}
\hline \multicolumn{3}{|c|}{ SIPHON } & \multicolumn{6}{|c|}{ SOIES DU SIPHON } & \multicolumn{3}{|c|}{ Mentum } \\
\hline A & & s & B & & $\mathrm{s}$ & C & D & E & Mt & & s \\
\hline 2,85 & \pm & 0,02 & 6,77 & \pm & 0,20 & 6,00 & 2,75 & 3,25 & 9,80 & \pm & 0,08 \\
\hline
\end{tabular}

\section{$3^{\circ}$ Caractères de Culex pipiens pipiens}

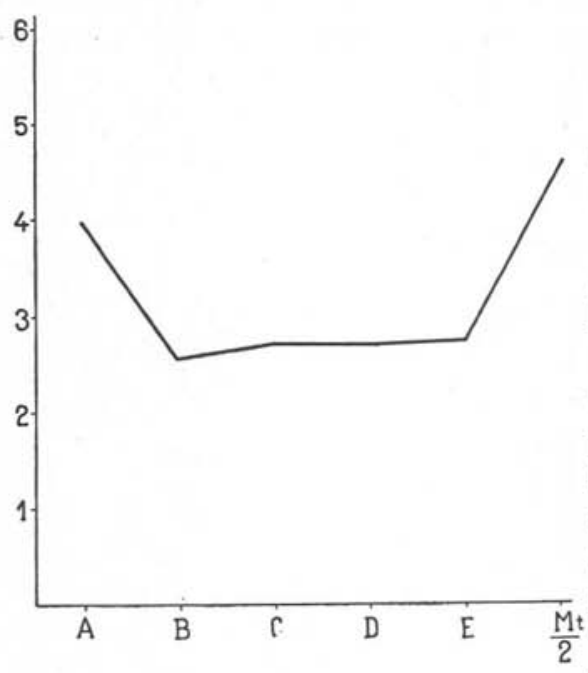

J'ai examiné à nouveau de nombreuses larves de Culex pipiens pipiens.

J'appelle ainsi des Culex obtenus à partir de grandes nacelles effilées aux deux extrémités (1), qui, à l'état adulte, sont de couleur sombre, à sternites abdominaux fortement marqués d'écailles noires, chez lesquels la trompe n'atteint pas l'extrémité distale du quatrième article du palpe mâle; qui sont absolument eurygames, anautogènes et ornithophiles.

Les larves de ces Culex vivent dans des eaux de sur-

Fı. 3. - Culex pipiens pipiens (environs de Strasbourg) face; elles sont caractérisées par l'indice siphonique élevé,

(1) Autant que j'ai pu en juger, la nacelle de $C$. fatigans, si elle est de grande taille elle aussi, n’est pas aussi effilée à ses extrén.ités que celle de C. p. pipiens. 
atteignant souvent 4 et le dépassant même parfois (sur exuvies) ; la faiblesse des indices B, C, D, E, et l'indice Mt inférieur à 10 . Je résume ces caractères dans le tableau III, tiré de différents échantillons et de diverses provenances, et dans la figure 3 :

Tablead III. - Larves de Culex pipiens pipiens

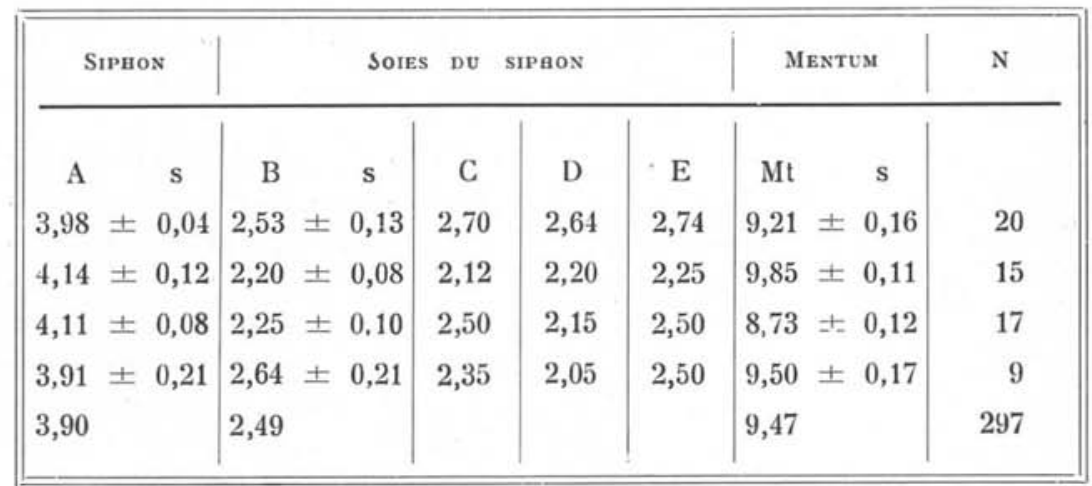

\section{Essais de croisements entre ces différents biotypes}

Le croisement le plus aisé à réaliser nous a paru être Culex $p$. autogenicus $q \times$ ơ C. p. fatigans; par contre, et Weyer, puis Roubaud l'avaient déjà vu, le croisement entre $C$. p. pipiens et $C$. p. fatigans est plus difficile à obtenir, au moins avec les souches européennes ; en Amérique, il semble qu'il en soit autrement à en juger par le travail récent de Rozeboom et Gilford.

\section{$1^{\circ}$ Groisement Culex autogenicus $q \times$ Culex fatigans}

Huit mâles de Culex fatigans sont placés en présence de neuf femelles de Culex autogenicus de la souche décrite ci-dessus, le 15 juin 1954, dans une cage de $30 \times 30 \times 30 \mathrm{~cm}$. Le 20 , huit pontes sont déposées par autogénèse. L'éclosion a lieu le 21 juin. Les larves s'élèvent facilement avec de la poudre de biscuit pour chiens. Il n'y a pas de mortalité anormale. Les nymphes apparaissent le 3 juillet et les mâles éclosent le 5 , les femelles le 7 juillet.

Les larves au quatrième stade présentent des caractères biométriques intermédiaires entre ceux des larves des parents, mais cependant les indices se rapprochent, tout au moins en ce qui concerne $\mathrm{B}$ et $\mathrm{C}$, de ceux des autogènes. Ils peuvent se résumer dans le tableau suivant (IV) et la figure 4 : 
Tableau IV. - Larves hybrides de $C$. aulogenicus $\$ \times \sigma^{\top}$ C. faligans, $\mathrm{F}^{4}$

\begin{tabular}{|c|c|c|c|c|c|c|c|c|c|c|}
\hline \multicolumn{3}{|c|}{ SiphoN } & \multicolumn{6}{|c|}{ SOIES DU SIPHON } & \multicolumn{2}{|c|}{ Mentum } \\
\hline A & & $\mathrm{s}$ & B & & $\mathrm{s}$ & $\mathrm{C}$ & D & E & Mit & $\mathbf{s}$ \\
\hline 3,10 & \pm & 0,03 & 4,43 & \pm & 0,15 & 4,10 & 2,50 & 2,90 & $9,95 \quad \pm$ & 0,08 \\
\hline
\end{tabular}

Les adultes issus de ces

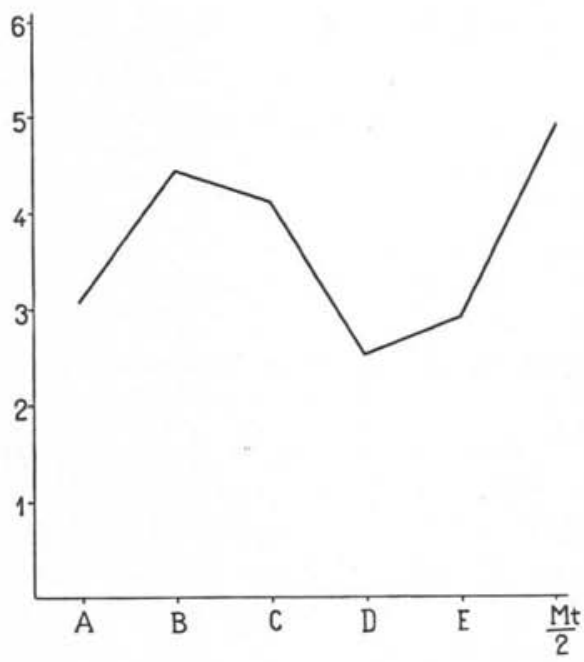

Fic. 4. - Hybrides (C. autogenicus $q \times$ C. fatigans $\left.\hat{o}^{3}\right)$ larves étaient de grande taille. L'ornementation abdominale est variable et la ponctuation sternale plus marquée cependant que chez les autogènes ; parfois, il existe deux petites taches triangulaires apicales d'écailles noires. Le mâle a un hypopygium intermédiaire qui rappelle les figures données autrefois par Weyer. La trompe du mâle dépasse toujours l'extrémité apicale du quatrième article des palpes.

Ces adultes sont ornithophiles, sténogames, non autogènes. Les femelles se gorgent facilement sur le poulet et donnent une seconde génération d'hybrides en se croisant avec les mâles homologues. La ponte est constituée par de grandes nacelles, rappelant celle des fatigans. Des œufs éclosent les 14 et 15 juillet.

Ces larves de seconde génération s'élèvent facilement. Leur morphogramme rappelle le précédent, mais cependant avec une sensible augmentation des indices $\mathrm{B}$ et $\mathrm{C}$, ce qui les rapproche du morphogramme théorique entre celui de larves de fatigans et d'autogenicus (tab. $\mathrm{V}$ et fig. 5). 
TABledU V. - Larves hybrides $C$. autogenicus $\odot \times \sigma^{7}$ C. fatigans $\mathrm{F}_{2}$

\begin{tabular}{|c|c|c|c|c|c|c|c|c|c|c|c|}
\hline \multicolumn{3}{|c|}{ SIPHON } & \multicolumn{6}{|c|}{ SOIES DU SIPHON } & \multicolumn{3}{|c|}{ Mentum } \\
\hline A & & s & B & & S & $\mathrm{C}$ & D & $\mathrm{E}$ & Mt & & $\mathbf{s}$ \\
\hline 3,05 & \pm & 0,02 & 4,80 & \pm & 0,2 & 4,50 & 2,85 & 2,90 & 10,30 & \pm & 0,13 \\
\hline
\end{tabular}

Les adultes de cette deuxiè-

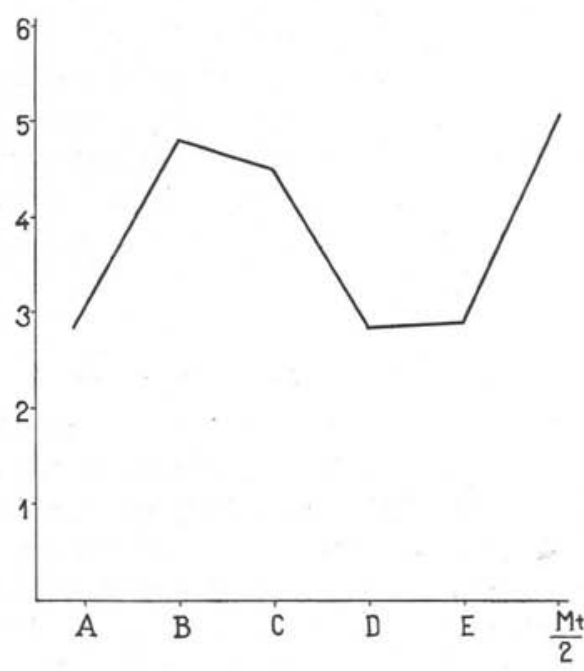
me génération sont semblables à ceux de la première, du moins en apparence. Sténogames et ornithophiles, ils se gorgent cependant facilement sur l'homme.

\section{Comportement DES ADULTES EN $F_{2}$. Autogénèse}

Mais alors que les adultes de $F_{1}$ ne présentèrent pas de trace d'autogénèse, le phénomène, comme cela se produit dans le croisement entre Mt $C$. pipiens autogenicus et C. pipiens pipiens, apparut à c e t $t$ e seconde génération. FIc. 5. - Hybrides (aut. $\uparrow \times$ fatig. $\left.\delta^{1}\right) \mathrm{F}_{2}$ Mais, cependant, j'insiste sur le fait qu'aucune ponte n'a été déposée grâce à ce processus ; par contre, il est visible par la dissection des femelles vierges ou fécondées, mais n'ayant pris aucune nourriture sanguine ou autre.

A l'examen de l'ovaire, le phénomène d'autogénèse est sensible et peut être affirmé lorsque la cellule germinale s'emplit de granulations et augmente de volume. L'image de ces œufs a été donnée pour le Culex autogenicus par Weyer en 1935 (1).

Des femelles de $\mathrm{F}_{2}$ sont donc placées dans les conditions requises

(1) Certains Culicides, qui ne sont certainement pas autogènes, peuvent présenter un léger piqueté de granulations dans la cellule germinale. J'ai pu voir de telles images chez des femelles vierges, non nourries, âgées de trois ou quatre jours, d'Anopheles maculipennis et d'Aedes cinerens. Ma:s ce n'est en rien comparable avec ce que l'on voit chez les autogènes. 
soit en présence de mâles, soit isolées, et vingt de ces femelles sont disséquées du troisième au sixième jour après l'éclosion.

Sur huit femelles disséquées, qui provenaient d'une cage contenant aussi des mâles, il est à remarquer que six n'étaient pas fécondées, ce qui est sans rapport avec le phénomène recherché, mais n'est pas sans intérêt. Quoi qu'il en soit, sur les vingt femelles disséquées, sept présentaient des signes évidents d'autogénèse et une femelle vierge, âgée de quatre jours, avait dans ses ovaires des œufs complètement formés. Mais, je le répète, aucune femelle non gorgée de sang, à cette génération, n'a déposé de ponte.

A la troisième génération d'hybrides, le phénomène ne fut pas plus apparent qu'à la génération précédente, c'est-à-dire qu'aucune ponte ne fut déposée par des femelles non gorgées. Mais la dissection de 30 femelles n'ayant absorbé aucune nourriture montra le phénomène d'autogénèse chez trois individus. Dans aucun ovaire, il n'y avait eu formation complète d'œufs, mais seulement un abondant passage de matériaux granuleux dans la cellule germinale qui occupait la moitié du volume de l'œuf.

\section{$2^{\circ}$ Groisement Culex p. fatigans $q \times \delta$ C. p. autogenicus}

Deux femelles vierges de $C$. fatigans s'étant nourries facilement sur l'homme sont mises dans une cage en présence de mâles autogènes au nombre de quatre ou cinq. Mais ces femelles, au lieu de former des œufs, deviennent vite maigres. Elles se regorgent plusieurs fois sur l'homme, sans résultat pour la ponte. Au bout d'une dizaine de jours, une de ces femelles est disséquée et on trouve les spermathèques pleines de spermatozoïdes, mais les œufs non développés avec à peine un léger piqueté dans les cellules germinales. L'autre femelle disséquée après vingt jours a des ovaires plus développés, un œuf unique est complètement formé ; mais les spermathèques sont vides...

Dans une autre expérience, aucune ponte n'est obtenue. Puis, dans une troisième série, j'obtiens une seule ponte fertile. Les femelles, cependant, avaient toutes été fécondées, mais il n’y avait pas eu de développement ovarien.

Les larves obtenues de la seule ponte fertile rappellent tellement celles des autogènes qu'il y avait dû y avoir erreur.

Il est difficile de tirer des conclusions de ces essais pas assez nombreux ; il en ressort que le sang humain ne peut toujours amener la maturation de certaines souches de $C$. fatigans et que l'étude de la nutrition de ce moustique au point de vue des affinités sanguines devrait être reprise. Le comportement de $C$. fatigans paraît 
différer sur ce point et d'autres encore suivant les régions; cela ressort aussi de ce qu'on trouve dans la littérature.

\section{$3^{\circ}$ Croisement entre C. p. fatigans et C. p. pipiens}

Huit mâles de Culex pipiens pipiens sont mis en présence de six femelles de $C$. pipiens fatigans dans une cage de $60 \times 60 \times 60 \mathrm{~cm}$., le 8 juillet, et la nourriture des femelles est assurée par le poulet. Deux femelles disséquées le 11 et le 13 ne sont pas fécondées. Les autres ne pondent pas et meurent.

Le 16 juillet, deux mâles de pipiens d'une autre souche sont placés dans une cage avec douze femelles de fatigans. Les femelles ne sont pas fécondées. Les autres ne pondent pas et meurent.

Le petit nombre d'essais ne permet pas de conclusions valables, mais il semble que ce croisement présente des difficultés avec les souches européennes et cela correspond avec ce qu'avaient vu Weyer et Roubaud.

\section{$4^{\circ}$ Groisement d'hybrides (C. autogenicus $\times$. C. fatigans) avec $C$. pipiens pipiens}

J'ai essayé de croiser $C$. pipiens pipiens avec un hybride $F_{1}$ d'autogenicus et de fatigans.

Une douzaine de mâles de $C$. pipiens pipiens sont introduits le 13 juillet dans une cage de $60 \times 60 \times 60 \mathrm{~cm}$. avec cinq femelles d'hybrides qui se gorgent facilement sur l'homme. Une ponte fertile est déposée le 19 juillet.

Le 31 juillet, les cinq femelles sont toujours vivantes, mais aucune nouvelle ponte n'ayant eu lieu elles sont toutes disséquées. L'ovaire paraît à l'état de repos et les spermathèques de trois femelles sont pleines de spermatozoïdes.

Les larves obtenues à partir de l'unique ponte ont des caractères biométriques moyens intermédiaires, mais les indices B, C, D, E, Mt sont plus élevés qu'il n'était prévisible (tab. VI, fig. 6) :

Tableav VI. - Larves issus d'hybrides $\sigma^{*} \times \odot C$. pipiens pipiens

\begin{tabular}{|c|c|c|c|c|c|c|c|c|c|c|}
\hline \multicolumn{3}{|c|}{ Siphox } & \multicolumn{6}{|c|}{ SoIEs de SIPHOX } & \multicolumn{2}{|c|}{ Mentes } \\
\hline A & & s & B & & $\mathrm{s}$ & C & D & $\mathrm{E}$ & Mt & $\mathrm{s}$ \\
\hline 3,34 & \pm & 0,03 & 4,35 & \pm & 0,32 & 3,90 & 2,78 & 2,82 & 10,16 & $\pm 0,16$ \\
\hline
\end{tabular}




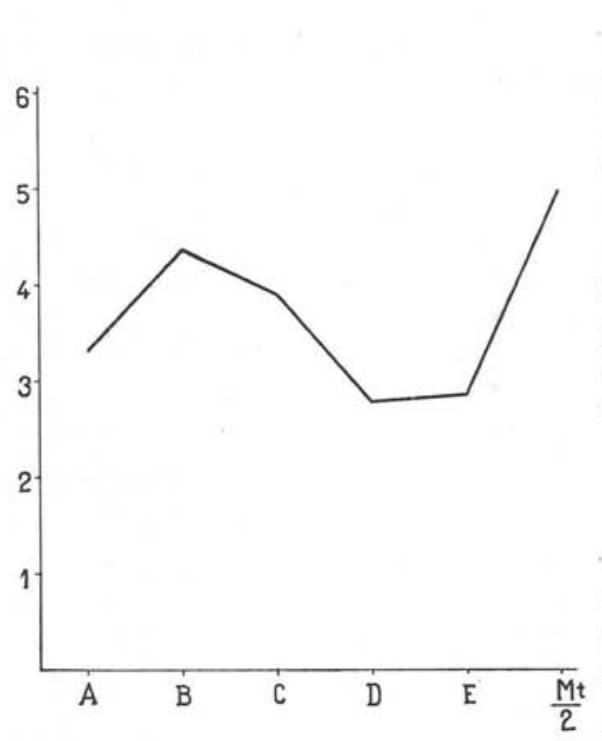

Les adultes obtenus à partir de ces larves ont un thorax foncé qui rappelle celui des pipiens et des sternites abdominaux dépourvus ou presque de ponctuation noire. Je n'ai malheureusement pu les suivre pour en étudier le comportement ou l'autogénèse.

\section{Conclusions}

Il ressort de ces expériences que le croisement entre Culex pipiens autogenicus femelle et le mâle de Culex pipiens fatigans est aisé à obtenir et que les larves FIr. 6. - Croisement hybrides o $\times$ C. p. pipiens hybrides ont un type intermerdiaire entre celui des parents.

La première génération d'hybrides ne présente pas le phénomène de l'autogénèse. La seconde l'a présenté, sans toutefois que (dans les limites de l'expérience) ce processus ait été suffisant pour amener la ponte.

Le croisement inverse (C. p. fatigans $+\times \delta^{t}$ C. p. autogenicus) semble plus délicat à réaliser, mais ceci tenait peut-être aux conditions de l'expérience.

Le croisement entre $C$. $p$. fatigans et $C$. $p$. pipiens n'a donné lieu qu'à peu de fécondations et aucune ponte fertile n'a été obtenue.

Le croisement entre hybrides $q$ (autogenicus $\times$ fatigans) et C. pipiens pipiens peut donner lieu à fécondation ; une ponte fertile obtenue a donné des larves ayant des caractères intermédiaires entre ceux des parents.

\section{BibLIOGRAPHIE}

On trouvera une abondante bibliographie sur le complexe Culex pipiens dans les rapports du symposium de la Société Entomologique de Londres :

Mattingly (P. F.) et coll. - The Culex pipiens complex. Trans. R. Entomol. Soc., CII, 1951 , p. $381-382$, fig. 
Callot (J.). - Etude sur quelques souches de Culex pipiens (sensu lato) et sur leurs hybrides. Ann. Parasitol., XXII, 1947, p. 380-387, fig.

Le rapport trompe/palpes dans les biotypes du complexe Culex pipiens et leurs hybrides. Ann. Parasitol., XXIX, 1954, p. 131-134.

FARID (M. A.). - Relationshịps between certain populations of Culex pipiens Linnaeus and Culex quinquefascialus Say in the United-States. Amer. Jl. Hyg., XLIX, 1949, p. 83-100, fig.

MAYr (E.), Linslex (E. G.) et Usinger (R. L.). - Methods and principles of systematic Zoölogy, New-York, 1952, 328 pp., fig.

Roubaud (E.). - Essai synthétique sur la vie du moustique commun, Culex pipiens. Ann. Sc. Nat., Zool., XVI, 1933, p. 5-168, fig.

Phénomènes d'amixie dans les intercroisements de Culicides du groupe pipiens. C.R. Ac. Sc., CCXII, 1941, p. 257.

Rоzевоoм (L. E.) et GLFord (B. N.). - Sexual isolation between populations of the Culex pipiens complex in North America. Jl. Parasitol., XL, 1954, p. 237-244.

Sunderaraman (S.). - Biometrical studies on intergradation in thr genitalia of certain populations of Culex pipiens and Culex quinquefasciatus in the United-States. Amer. Jl. Hyg., L, 1949, p. 237-244.

Weyer (F.). - Die Rassenfrage bei Culex pipiens in Deutschland. Zeitschr. f. Parasitenk., VIII, 1935, p. 104-115, fig.

Kreuzungsversuche bei Stechmücken. Arb. phys. angew. Entom., Berlin Dahlem, III, 1936, p. 202-228, fig.

(Institut de Parasitologie, Faculté de Médecine de Strasbourg, Prof. J. Callot) 